\title{
Front Matter: Volume 9915
}

, "Front Matter: Volume 9915," Proc. SPIE 9915, High Energy, Optical, and Infrared Detectors for Astronomy VII, 991501 (5 October 2016); doi: $10.1117 / 12.2254994$

SPIE Event: SPIE Astronomical Telescopes + Instrumentation, 2016, Edinburgh, SPIE. United Kingdom 


\title{
PROCEEDINGS OF SPIE
}

\section{High Energy, Optical, and Infrared Detectors for Astronomy VII}

\author{
Andrew D. Holland \\ James Beletic \\ Editors
}

26-29 June 2016

Edinburgh, United Kingdom

Sponsored by

SPIE

Cooperating Organizations

American Astronomical Society (United States) - Australian Astronomical Observatory (Australia) - Association of Universities for Research in Astronomy (AURA) - Canadian Astronomical Society (CASCA) (Canada) • Canadian Space Agency (Canada) • European Astronomical Society (Switzerland) • European Southern Observatory (Germany) • National Radio Astronomy Observatory • Royal Astronomical Society (United Kingdom) • Science \& Technology Facilities Council (United Kingdom)

Published by

SPIE

\section{Volume 9915}

Part One of Two Parts 
The papers included in this volume were part of the technical conference cited on the cover and title page. Papers were selected and subject to review by the editors and conference program committee. Some conference presentations may not be available for publication. The papers published in these proceedings reflect the work and thoughts of the authors and are published herein as submitted. The publisher is not responsible for the validity of the information or for any outcomes resulting from reliance thereon.

Please use the following format to cite material from these proceedings:

Author(s), "Title of Paper," in High Energy, Optical, and Infrared Detectors for Astronomy VII, edited by Andrew D. Holland, James Beletic, Proceedings of SPIE Vol. 9915 (SPIE, Bellingham, WA, 2016) Article CID Number.

ISSN: 0277-786X

ISBN: 9781510602090

ISSN: 1996-756X (electronic)

ISBN: 9781510602106 (electronic)

Published by

SPIE

P.O. Box 10, Bellingham, Washington 98227-0010 USA

Telephone +1 3606763290 (Pacific Time) · Fax +1 3606471445

SPIE.org

Copyright (C) 2016, Society of Photo-Optical Instrumentation Engineers.

Copying of material in this book for internal or personal use, or for the internal or personal use of specific clients, beyond the fair use provisions granted by the U.S. Copyright Law is authorized by SPIE subject to payment of copying fees. The Transactional Reporting Service base fee for this volume is $\$ 18.00$ per article (or portion thereof), which should be paid directly to the Copyright Clearance Center (CCC), 222 Rosewood Drive, Danvers, MA 01923. Payment may also be made electronically through CCC Online at copyright.com. Other copying for republication, resale, advertising or promotion, or any form of systematic or multiple reproduction of any material in this book is prohibited except with permission in writing from the publisher. The CCC fee code is 0277-786X/16/\$18.00.

Printed in the United States of America.

Publication of record for individual papers is online in the SPIE Digital Library.

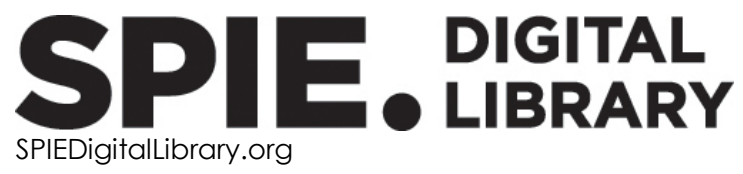

Paper Numbering: Proceedings of SPIE follow an e-First publication model. A unique citation identifier (CID) number is assigned to each article at the time of publication. Utilization of CIDs allows articles to be fully citable as soon as they are published online, and connects the same identifier to all online and print versions of the publication. SPIE uses a six-digit CID article numbering system structured as follows:

- The first four digits correspond to the SPIE volume number.

- The last two digits indicate publication order within the volume using a Base 36 numbering system employing both numerals and letters. These two-number sets start with 00, 01, 02, 03, 04, 05, 06, 07, 08, 09, OA, OB ... 0Z, followed by 10-1Z, 20-2Z, etc. The CID Number appears on each page of the manuscript. 


\title{
Contents
}

\author{
ix Authors \\ $\mathrm{xv}$ Conference Committee \\ xvii Introduction
}

\section{Part One}

\section{SESSION 1 DETECTOR PROGRAMMES}

991502 The status of European Space Agency supported detector developments [9915-1]

991504 e2v CCD and CMOS sensors and systems designed for astronomical applications [9915-3]

991505 Leonardo (formerly Selex ES) infrared sensors for astronomy: present and future [9915-4]

\section{SESSION 2 EM CCDS}

991506 Development of the x-ray camera for the OGRE sub-orbital rocket [9915-5]

991507 The faint intergalactic-medium red-shifted emission balloon: future UV observations with EMCCDs [9915-6]

991508 The effect of proton radiation on the EMCCD for a low Earth orbit satellite mission [9915-7]

9915 OA Cryogenic irradiation of an EMCCD for the WFIRST coronagraph: preliminary performance analysis [9915-242]

\section{SESSION $3 \quad$ IR SENSORS}

9915 OB MCT APD focal plane arrays for astronomy at CEA-LETI [9915-9]

9915 OC Development activities on NIR large format MCT detectors for astrophysics and space science at CEA and SOFRADIR [9915-10]

9915 OD Candidate 10 micron HgCdTe arrays for the NEOCam space mission [9915-11]

9915 OE 13 micron cutoff $\mathrm{HgCdTe}$ detector arrays for space and ground-based astronomy [9915-12]

9915 OF Performance of science grade HgCdTe H4RG-15 image sensors [9915-13]

9915 OG Mitigation of H2RG persistence with image illumination [9915-14] 
$9915 \mathrm{OH} \quad$ Random telegraph signal (RTS) noise and other anomalies in the near-infrared detector systems for the Euclid mission [9915-20]

99150 Mapping radiation-induced defects in CCDs through space and time [9915-16]

$99150 \mathrm{~J}$ A study of the double-acceptor level of the silicon divacancy in a proton irradiated $\mathrm{n}$ channel CCD [9915-15]

9915 OK Radiation effects on the Gaia CCDs after 30 months at L2 [9915-17]

$9915 \mathrm{OL}$ Charge transfer efficiency in a p-channel CCD irradiated cryogenically and the impact of room temperature annealing [9915-18]

$99150 \mathrm{M}$ The effect of radiation-induced traps on the WFIRST coronagraph detectors [9915-19]

SESSION $5 \quad$ IR DETECTORS I

9915 ON Next-generation performance of SAPHIRA HgCdTe APDs [9915-22]

991500 Towards the next generation of L-APD MOVPE HgCdTe arrays: beyond the SAPHIRA $320 \times 256[9915-21]$

9915 OP New progress in electron-injection detectors for NIR imagers with low noise and high frame rates [9915-23]

$99150 Q \quad$ RVS WFIRST sensor chip assembly development results [9915-24]

9915 OR Progress on the characterization activities of new infrared detectors from Leonardo, UK at the UKATC [9915-25]

\section{SESSION 6 CCDS}

9915 OS Status report on STA detectors and electronics for 2016 [9915-27]

$99150 U$ Technology validation of the PLATO CCD at ESA [9915-29]

$99150 \mathrm{~V}$ Characterization and acceptance testing of fully depleted thick CCDs for the large synoptic survey telescope [9915-30]

\section{SESSION 7 TEST AND CHARACTERISATION}

9915 0W Performance of the first science grade $\lambda c=2.5 \mu \mathrm{m}$ HAWAll 4RG -15 array in the laboratory and at the telescope [9915-31]

9915 OX Integrated system tests of the LSST raft tower modules [9915-32] 
9915 OY Development of low-noise CCD drive electronics for the world space observatory ultraviolet spectrograph subsystem [9915-33]

9915 OZ Optical and dark characterization of the PLATO CCD at ESA [9915-34]

991510 On-ground characterization of the Euclid's CCD273-based readout chain [9915-35]

991511 Performance overview of the Euclid infrared focal plane detector subsystems [9915-114]

\section{SESSION 8 CMOS IMAGE SENSORS}

991512 LGSD/NGSD: high speed visible CMOS imagers for E-ELT adaptive optics [9915-36]

991513 Fully depleted and backside biased monolithic CMOS image sensor [9915-38]

991514 A 9 megapixel large-area back-thinned CMOS sensor with high sensitivity and high framerate for the TAOS II program [9915-39]

991515 Electro-optic and radiation damage performance of the CIS115, an imaging sensor for the JANUS optical camera onboard JUICE [9915-40]

\section{SESSION 9 QE IMPROVEMENTS AND CALIBRATION}

991517 High accuracy measurements of the intrapixel sensitivity of VIS to LWIR astronomical detectors: experimental demonstration [9915-42]

99151 A High fidelity point-spread function retrieval in the presence of electrostatic, hysteretic pixel response [9915-45]

SESSION 10 CMOS X-RAY SENSORS

9915 1D The HEXITEC hard x-ray pixelated CdTe imager for fast solar observations [9915-50]

\section{Part Two}

SESSION 11 IR DETECTORS II

$9915 \mathrm{IE}$ Comparison of persistence in spot versus flat field illumination and single pixel response on a Euclid HAWAII-2RG at ESTEC [9915-51]

$99151 \mathrm{G}$ Large format array NIR detectors for future ESA astronomy missions: characterization and comparison [9915-53]

$99151 \mathrm{H} \quad$ Flexible focal plane arrays for UVOIR wide field instrumentation [9915-55] 
$99151 \mathrm{~J} \quad$ Electron multiplying CMOS as Shack-Hartmann wavefront sensor [9915-37]

$99151 \mathrm{~K} \quad$ Shutter heating system of Antarctic bright star survey telescope [9915-56]

$99151 \mathrm{~L}$ Scientific CCD controller for the extreme environment at Antarctic [9915-57]

$99151 \mathrm{M} \quad$ Back-illuminated large area frame transfer CCDs for space-based hyper-spectral imaging applications [9915-58]

$99151 \mathrm{~N} \quad$ Large format array controller (aLFA-C): tests and characterisation at ESA [9915-59]

991510 Low temperature performance of a commercially available InGaAs image sensor [9915-60]

$99151 \mathrm{P} \quad$ Noise optimization of the source follower of a CMOS pixel using BSIM3 noise model [9915-61]

$99151 Q \quad$ Measuring extended red sensitivity in a 1.7um-cutoff HgCdTe detector array [9915-62]

9915 is DCDS weighted averaging theory and development for improved noise filtering in scientific CCD applications [9915-64]

9915 1T NUV performance of e2v large BICMOS array for CASTOR [9915-66]

$99151 \mathrm{U}$ Infrared APD characterization at NRC [9915-67]

9915 IW NIR camera and spectrograph SWIMS for TAO 6.5m telescope: array control system and its performance [9915-69]

$99151 Y$ Characterization of H2RG IR detectors for the Euclid NISP instrument [9915-71]

991520 Evaluating noise performance of the IUCAA sidecar drive electronics controller (ISDEC) based system for TMT on-instrument wavefront sensing (OIWFS) application [9915-73]

991521 ESA's CCD test bench for the PLATO mission [9915-75]

991522 Experiments with synchronized sCMOS cameras [9915-76]

991523 A project plans to develop two ASICs for CCD controller [9915-77]

991524 IDSAC-IUCAA digital sampler array controller [9915-78]

991525 Measuring the effective pixel positions for the HARPS3 CCD [9915-79]

991526 ISDEC-2 and ISDEC-3 controllers for HAWAll detectors [9915-80]

991528 Silicon photomultipliers as readout elements for a Compton effect polarimeter: the COMPASS project [9915-82] 
991529 Comparing simulations and test data of a radiation damaged CCD for the Euclid mission [9915-83]

9915 2A Simplified charge transfer inefficiency correction in CCDs by trap-pumping [9915-84]

9915 2B MeV-level electron and gamma ray sensitivites of modern far ultraviolet sensitive microchannel plate detectors [9915-85]

$99152 \mathrm{C}$ Further performance evaluation of $5.3 \mu \mathrm{m}$ cut-off Hawaii-2RG detectors [9915-86]

9915 2D A novel reflectometer for relative reflectance measurements of CCDs [9915-87]

$99152 \mathrm{G}$ Second generation large area microchannel plate flat panel phototubes [9915-91]

$99152 \mathrm{H} \quad$ Positional calibrations of the germanium double sided strip detectors for the Compton spectrometer and imager [9915-92]

9915 2l Signal dependence of inter-pixel capacitance in hybridized HgCdTe H2RG arrays for use in James Webb space telescope's NIRcam [9915-93]

$99152 \mathrm{~J} \quad$ Design and development of hard x-ray imaging detector using scintillator and $\mathrm{Si}$ photomultiplier [9915-94]

9915 2K A generic FPGA-based detector readout and real-time image processing board [9915-95]

9915 2L Characterization of an ultraviolet imaging detector with high event rate ROIC (HEROIC) readout [9915-96]

$99152 \mathrm{M} \quad$ Radiation testing of CID arrays [9915-97]

9915 2N Characterizing persistence in JWST NIRCam flight detectors [9915-98]

991520 CCD emulator design for LSST camera [9915-100]

9915 2P A microwave kinetic inductance detector for the DAG telescope [9915-101]

$99152 \mathrm{Q}$ Single event effects in $0.18 \mu \mathrm{m}$ CMOS image sensors [9915-102]

9915 2T Front-end and slow control electronics for large area SiPMs used for the single mirror Small Size Telescope (SST-1M) of the Cherenkov Telescope Array (CTA) [9915-105]

$99152 \mathrm{U}$ Persistence characterization and data calibration scheme for the RSS-NIR H2RG detector on SALT [9915-106]

$99152 \mathrm{~V}$ Detector control and data acquisition for the wide field infrared survey telescope (WFIRST) with a custom ASIC [9915-107]

$99152 X \quad$ RVS large format arrays for astronomy [9915-109]

$99152 Y \quad$ Low voltage electron multiplying CCD in a CMOS process [9915-111]

$99152 Z$ A temperature controller board for the ARC controller [9915-112] 
991530 Implementation of an FPGA-based DCDS video processor for CCD imaging [9915-113]

Proc. of SPIE Vol. $9915991501-8$

Downloaded From: https://www.spiedigitallibrary.org/conference-proceedings-of-spie on 26 Apr 2023 Terms of Use: https://www.spiedigitallibrary.org/terms-of-use 


\title{
Authors
}

Numbers in the index correspond to the last two digits of the six-digit citation identifier (CID) article numbering system used in Proceedings of SPIE. The first four digits reflect the volume number. Base 36 numbering is employed for the last two digits and indicates the order of articles within the volume. Numbers start with 00, 01, 02, 03, 04, 05, 06, 07, 08, 09, OA, OB...0Z, followed by 10-12, 20-2Z, etc.

\author{
Abreu, Asier, OK \\ Acharya, Y. B., 2J \\ Aguilar, J. A., 2T \\ Aliş, Sinan, $2 P$ \\ Alkire, Greg, 2V \\ Allanwood, E. A. H., 15 \\ Alvarez, Domingo, 2C \\ Amman, M., 2H \\ Antilogus, Pierre, 0X, 1A \\ Arkun, Erdem, OF \\ Astier, Pierre, $1 \mathrm{~A}$ \\ Atkinson, Dani E., ON, OW \\ Atkinson, David, OR \\ Auyeung, John, OF \\ Azzollini, R., 10 \\ Baker, Ian M., 05, ON, 0O, OR \\ Barbier, Rémi, $\mathrm{OH}, 11,1 \mathrm{E}, 1 \mathrm{~J}, 1 \mathrm{Y}$ \\ Barkhouser, Robert H., 2D \\ Barnes, Keith, 05, OR \\ Baum, Stefi, 21 \\ Baumgartner, Wayne H., ID \\ Beam, Carey, $2 \mathrm{M}$ \\ Beaton, Alexander, $1 \mathrm{~T}$ \\ Beaufort, Thierry, OK, OU, OZ, 1E, 1G, 21 \\ Beckmann, Udo, 2C \\ Beletic, James W., OF \\ Bender, Chad, IQ \\ Beuville, Eric, $2 X$ \\ Bezawada, Naidu, OR \\ Bharti, Rupali, 26 \\ Bhaskaran, Suraj, 2M \\ Billotta, S., 28 \\ Bilnik, W., 2T \\ Blank, Richard, OW \\ Blase, Ryan C., 2B \\ Blocki, J., 2T \\ Bloemmaert, Sander, 0U, 0Z, 1E, 1G, 1N, 21 \\ Boe, Raymond, $2 \mathrm{X}$ \\ Bogacz, L., 2T \\ Boggs, Kasey, OS \\ Boggs, S., $2 \mathrm{H}$ \\ Bonnefois, A., 17 \\ Borkowski, J., 2T \\ Boulade, Olivier, OB, OC, 17 \\ Bradley, Scott, $2 \mathrm{~L}$ \\ Brandonisio, A., 28 \\ Bredthaver, Gregory, OS \\ Bryson, lan, OR \\ Bugnet, Henri, 2Q
}

Bulik, T., 2T

Burgon, Ross, OA

Burse, Mahesh P., 20, 24, 26

Burt, D., OL

Bush, Nathan, OA, OI, OM

Butler, Bart, OU, OZ, 1E, 1G, 1N, 21

Buton, C., $1 \mathrm{~J}$

Cabrera, Mario S., OD, OE

Cadoux, F., 2T

Cagiano, S., 11

Caillat, Laurence, 1 Y

Carmody, Micheal, OF

Castelein, Pierre, $\mathrm{OC}$

Catalano, O., 28

Cervera, Cyril, OC

Chambion, Bertrand, $1 \mathrm{H}$

Chang, H. K., 2H

Chattopadhyay, Sabyasachi, 20, 24

Chen, J., 11

Chen, Jie, 1K, $1 \mathrm{~L}$

Cheng, Edward, OQ, 2V

Cheung, S., 11

Chiang, Jim, OV

Chillal, Kalpesh, 24, 26

Chiu, J. L., 2H

Cho, H., 11

Chordia, Pravin, 20, 24, 26

Chorier, Philippe, OC

Christe, Steven D., ID

Christov, A., 2T

Ciapponi, Alessandra, 02

Cillis, A., 11

Clapp, Matthew, OY, is

Clarke, Andrew S., 13

Clémens, Jean-Claude, $\mathrm{OH}, 11,1 Y$

Content, David, OQ, 2V

Conversi, Luca, $\mathrm{OH}$

Cooke, Chathan M., 2B

Copperwheat, Chris M., 22

Cordrey-Gale, Matthew, $2 Q$

Corrales, Elizabeth, $0 Q, 2 X$

Costa, E., 28

Côté, Patrick, $1 \mathrm{~T}$

Craven, C. A., 2G

Cropper, M., 10

Crouzet, Pierre-Elie, 02, 1E, $1 \mathrm{G}$

Crowley, Cian, OK

Culver, Harry, 2V

Curyło, M., 2T 
Cutts, A., 10

Daigle, Olivier, 08

Davis, Michael W., 2B

Davis, Ray, OR

Dawson, O., 11

de Borniol, E., OB

de la Barrière, F., 17

De Ridder, J., $1 \mathrm{P}$

Defernez, Arnaud, 12

Del Monte, E., 28

Delisle, C., 17

della Volpe, D., 2T

Delo, G., 11

Demers, Richard, OA, OM

DeRoo, Casey T., 06

Devashrayee, Niranjan M., 2J

Di Cosimo, S., 28

Di Persio, G., 28

Dierickx, Bart, 12

Doherty, Peter, OX, 1A

Dong, Shucheng, IK

Donlon, Kevan, 21

Dorn, Meghan L., OD, OE

Downing, Mark, 12

Doyle, Christy, $2 \mathrm{X}$

Drab, John, 2X

Druart, G., 17

Dryer, B., OL

Dubreuil, D., 17

Dunford, Alice, $2 Y$

Duvet, Ludovic, 02, 0U, 0Z, 1E, 1G, 1N, 21

Dyrda, M., 2T

Ealet, Anne, $\mathrm{OH}, 1 \mathrm{Y}$

Ebbets, Dennis, $2 \mathrm{~L}$

Edwall, Dennis, OF

Effinger, Robert, OM

Eggen, Nathan, $2 \mathrm{U}$

Endicott, James, $2 Q$

Erol, Ayşe, 2P

Ertley, C. D., 2G

Fabiani, S., 28

Farris, Mark, OF, OW, 11

Fathipour, Vala, OP

Favre, Y., 2T

Feautrier, Philippe, OB, 12

Feizi, A., 11

Feng, Yi, $1 \mathrm{~L}$

Fereyre, P., 1J

Ferrando, P., 10

Ferrari, Marc, $1 \mathrm{H}$

Ferriol, Sylvain, $\mathrm{OH}, 1 \mathrm{Y}$

Fièque, Bruno, $\mathrm{OC}$

Finger, Gert, 0O, 2C

Fodness, Bryan, $2 M$

Foley, M. R., $2 \mathrm{G}$

Foltz, R., 11

Forrest, William J., OD, OE

Fournier, M., $1 \mathrm{~J}$

France, Kevin, 2L

Franka, Steve, 2L
Frankowski, A., 2T

Freymiller, Ed, 2L

Fried, J., 20

Fryer, Martin, 12

Fulk, Chad, OQ, 2X

Gach, Jean-Luc, 12

Gaeremynck, Yann, 1H

Gaschet, Christophe, 1H

Gaskin, Jessica, 1D

Geimer, Christoph, 2C

Getin, Stéphane, 1H

Getty, Jonathan, 0Q, 2X

Giarrusso, S., 28

Gillard, William, $1 Y$

Gilmore, Angelo S., 1M

Gilmore, Kirk, 1A

Gladstone, G. Randall, 2B

Goebel, Sean B., ON, OW

Gooding, David, 1E, $1 \mathrm{G}$

Gow, Jason P. D., OA, Ol, OJ, OL, 15, 29, 2A

Goyal, S. K., 2J

Gravrand, Olivier, OB, OC

Greathouse, Thomas K., 2B

Gregory, Kyle, 1D

Grudniki, Ł., 2T

Grudzinska, M., 2T

Guellec, Fabrice, 0C

Guérineau, N., 17

Gunn, James E., OF, 2D

Güver, Tolga, 2P

Guyon, Olivier, OP

Guyonnet, Augustin, 1A

Hailey, M., 10

Hall, David J., 06, OA, OI, OJ, OL, OM, 29

Hall, Donald N. B., OF, ON, 0O, OW

Hall, Richard D., 25

Halverson, Samuel P., $1 Q$

Hamden, Erika T., 07

Harding, Leon K., OA, OM

Hardy, Tim, $1 \mathrm{U}$

Hart, Murdock, 2D

Harwit, Alex, 2L

Haupt, Justine, OV, OX

Hayes-Thakore, Chris, OY

Heijnen, Jerko, OU, OZ, 1E, 1G, 1N, 21

Heininger, Matthias, 2C

Heller, M., 2T

Henry, David, $1 \mathrm{H}$

Herrmann, S., OX

Hickey, M., 11

Hill, Robert J., OQ

Hipwood, Les, 05, OR

Hodapp, Klaus W., OF, OW

Hoenk, Michael, OA

Holland, Andrew D., 06, OA, OI, 0J, OL, 13, 15, 29,

2Q, $2 Y$

Holland, Eric, OF

Holland, Karen, 06

Holmes, W., 11

Hu, D., 10 
Hudson, Danya, 08

Huffer, M., OX

Hugot, Emmanuel, $1 \mathrm{H}$

Hunt, T., 10

Hutchings, John, $1 \mathrm{~T}$

Hwang, T., 11

Idźkowski, B., 2T

Impiombato, D., 28

Inglis, Andrew R., ID

Israelsson, U., 11

Ives, Derek, 2C

Jackson, Malcolm, OR

Jacobson, Shane M., ON, OW

Jaehnig, Kurt, $2 \mathrm{U}$

Jahn, Wilfried, $1 \mathrm{H}$

Jamrozy, M., 2T

Janiak, M., 2T

Jean, $\mathrm{P}$., $2 \mathrm{H}$

Jelinsky, S. R., 2G

Jermak, Helen, 22

Jerram, Paul, 04, 12, 14

Jhabvala, M., 11

Jiang, Fengxin, $1 \mathrm{~K}$

Johnson, Steven, 14

Jordan, Douglas, 04, 0A

Jorden, Paul, 04, 12, 14

Joshi, Atul, 2V

Joshi, Bhushan, 24

Juramy-Giles, C., OX

Kahle, D., 11

Kan, Em., 11

Kan, Er., 11

Kashikawa, Nobunari, 10

Kasperek, J., 2T

Kato, Natsuko M., IW

Kay, Burak, 2P

Kelly, Daniel, 2V

Kern, P., OB

Keskin, Onur, 2P

Ketchazo, C., 17

Khalil, A., 10

Kierans, C., $2 \mathrm{H}$

Kilcoyne, Sean, $2 X$

King, James, OY

Kitagawa, Yutaro, IW

Kobayakawa, Yutaka, IW

Kohley, Ralf, OH, OK, 10, $1 \mathrm{E}$

Kohok, Abhay, 20, 26

Kolb, Johann, 12

Komiyama, Yutaka, 10

Konishi, Masahiro, IW

Kotov, Ivan V., OV, 1 A

Kotulla, Ralf, $2 \mathrm{U}$

Kubik, Bogna, $\mathrm{OH}, 1 Y$

Kuczewski, J., OX, 20

Kyne, Gillian, 07

Ladiya, T., 2J

Lage, Craig, $1 \mathrm{~A}$

Lagier, Philippe, $1 Y$

Lalik, K., 2T
Le Bouquin, J.-B., OB

Leese, M., 15

Legras, Romain, $1 Y$

Lehner, Matthew, 14

Leisenring, Jarron M., $2 \mathrm{~N}$

Lemmel, Frédéric, OU, OZ, 1E, 1G, 1N, 21

Levillain, Yves, 02

Lewis, Matthew R. F., 06

Lin, C. H., $2 \mathrm{H}$

Lin, Sheng-zhao, $1 \mathrm{~L}$

Lingner, Nicole, 07

Lockhart, Charles, $0 \mathrm{~N}$

Loose, Markus, OW, 11, 2V

Lotkin, G., 11

Lowell, A., $2 \mathrm{H}$

LU, W., $2 \mathrm{O}$

Luppino, Gerard, 2V

Lupton, Robert, 1A

Lyard, E., 2T

Lyashenko, A. V., $2 \mathrm{G}$

Mach, E., 2T

Maciaszek, Thierry, $1 Y$

Mah, Jonathan, $0 Q, 2 \mathrm{~V}$

Mahadevan, Suvrath, IQ

Mahato, Swaraj, IP

Mainzer, Amy K., OD

Mandat, D., 2T

Marszalek, A., 2T

Martin, D. Christopher, 07

Massaro, E., 28

Mathew, Joice, 2K

Maxey, Chris, 05

Mayer, Frederic, 1 J, 2Q

Mazin, Benjamin A., 2P

McBride, S., 2H

McEntaffer, Randall L., 06

McLeod, Brian A., OG

McLevige, William, OF

MCMurtry, Craig W., OD, OE

Mears, Lynn, OQ, 2X

Mehrgan, Leander, 2C

Mestri, Vilas, 26

Meyer, Manfred, 12

Meynants, Guy, $1 \mathrm{P}$

Meynart, Roland, 02

Michaels, Darren, OA

Michalowski, J., 2T

Michilli, D., 28

Mihalcea, lonut, OY, IS

Miko, Laddawan, 11, 2V

Miller, Chris, OR

Mineo, T., 28

Minoglou, Kyriaki, 02

Minot, M. J., 2G

Misselt, Karl, 2N

Miyata, T., 27

Moderski, R., 2T

Mohseni, Hooman, OP

Monson, Andrew J., 1Q

Montaruli, T., 2T 
Morbidini, A., 28

Moreau, Vincent, 0C, 17

Morelli, E., 28

Morrissey, Patrick, 07, 0M

Morse, Tom, OY, $1 \mathrm{~S}$

Mosby, Gregory, 2U

Motohara, Kentaro, IW

Moulin, Gaid, 1H

Mugnier, L., 17

Muleri, F., 28

Mulet, Patrick, 0C, 17

Murray, Neil J., 06, 0A, OI, OL, 29, 2A

Murthy, Jayant, 2K

N.P. S., Mithun, 2J

Nagayama, Takahiro, 10

Naik, Amisha P., 2J

Nakaya, Hidehiko, 10

Neal, Homer, OV

Nell, Nicholas, 2L

Nelms, Nick, 02, $1 \mathrm{~N}$

Nelson, David, $\mathrm{OQ}$

Nemati, Bijan, OA, OM

Neronov, A., 2T

Nguyen, L., 11

Niclas, Mathieu, 1 Y

Niemiec, J., 2T

Nikitushkina, Liubov, $1 \mathrm{H}$

Nikzad, Shouleh, 07

Ninkov, Zoran, 2I, 2M

Nomerotski, Andrei, 1A

Norup Sorensen, Anton, $1 Y$

O'Mahony, A., 2G

O'Brien, Kieran, 2P

O'Connor, Paul, OV, 0X, 1A, 20

Ohashi, Hirofumi, IW

Oosterbroek, Tim, OU, OZ

Ostrowski, M., 2T

Pacciani, L., 28

Park, Min-Su, OP

Paśko, P., 2T

Patel, A. R., 2J

Pech, M., 2T

Peddada, Pavani, OA

Peralta, Richard, 2X

Philbrick, Robert H., $1 \mathrm{M}$

Piche, Louis, 08

Pike, Andrew, 12

Pipher, Judith L., OD, OE

Piquette, E., 11

Plana, C., 10

Popecki, M., 2G

Porcelli, A., 2T

Poshyachinda, Saran, 22

Pottinger, S., 10

Powers, T., 11

Prabhudesai, Swapnil, 20

Prandini, E., 2T

Pratlong, Jérôme, 04, 14, 15

Pravdo, S., 11

Prieto, Eric, $1 Y$
Prod'homme, Thibaut, 02, 0K, OU, 0Z, 1G, 21, 29

Punnadi, Sujit, 20, 26

Queloz, Didier, 25

Rajda, P., 2T

Ramaprakash, A. N., 20, 24, 26

Rameez, M., 2T

Ramsey, Larry, $1 Q$

Raskin, Gert, 1P

Rasmussen, Andrew, IA

Retherford, Kurt D., 2B

Reyes, Javier, 12

Rezaei, Mohsen, OP

Rieke, Marcia, $2 \mathrm{~N}$

Robberto, Massimo, 2N

Robbins, Mark, 04

Rossetti, Dino, 2V

Rothman, J., OB

Roumegoux, Julien, OC

Roy, Niladri, $1 \mathrm{~T}$

Rubini, A., 28

Runkle, A., 11

Rushton, Joseph E., 2Q

Russo, S., OX

Ryan, Daniel F., ID

Safonova, Margarita, 2K

Salter, Mike, OY, IS

Sarpotdar, Mayuresh, 2K

Schioppa, E. jr., 2T

Schmoll, J., 10

Schovanek, P., 2T

Schrein, Ronald J., $1 \mathrm{M}$

Schultz, Ted B., 06

Scott, Alan, 08, $1 T$

Secroun, Aurélia, $\mathrm{OH}, 1 \mathrm{Y}$

Seiffert, M., 11

Seller, Paul, 1D

Serra, Benoit, $1 Y$

Seweryn, K., 2T

Sharples, R., 10

Shih, Albert Y., 1D

Shimono, Atsushi, OF

Shorrocks, Nick, OR

Siegmund, O. H. W., $2 \mathrm{G}$

Sinha, Sakya, 20, 26

Siskind, Eric, $2 \mathrm{~V}$

Skottfelt, Jesper, OI, 0M, 29

Skowron, K., 2T

Sleator, C., $2 \mathrm{H}$

Sliusar, V., 2T

Smadja, Gérard, $1 Y$

Smee, Stephen A., OF, 2D

Smit, Hans, OU, OZ, 1E, 1G, 1N, 21

Smith, Brian, 2V

Smith, D. R, 15

Smith, Ken, 08

Smith, Robert J., 22

Smith, Roger, OG

Smith, Thomas, OV

Soffitta, P., 28

Soman, Matthew R., 06, 15 
Song, Qian, 23

Soonthorntham, Boonrucksar, 22

Sottile, G., 28

Sowinski, M., 2T

Stadler, Eric, 12

Starr, Barry, OQ, $2 X$

Stawarz, Ł., 2T

Steele, lain A., 22

Stefanov, Konstantin D., 13, 15, 2Q, $2 Y$

Stodulska, M., 2T

Stodulski, M., 2T

Strada, Paolo, $\mathrm{OH}, 11,1 \mathrm{E}$

Stubbs, Christopher, 0X, 1A

Sun, Quan, 23

Swift, Nick, 12

Szafraniec, Magdalena, 10

Takacs, Peter, OV

Takahashi, Hidenori, IW

Tamura, Naoyuki, OF

Tan, Chee Leong, OP

Tateuchi, Ken, $1 \mathrm{~W}$

Tedesco, J., $2 \mathrm{G}$

Ter Haar, Joerg, 1E, 1G, IN

Terao, Yasunori, IW

Terrien, Ryan C., 1Q

Theobald, C., 10

Thompson, Samantha, 25

Tilquin, André, $1 Y$

Todo, Soya, IW

Tomsick, J., $2 \mathrm{H}$

Toscano, S., 2T

Tracy, Christopher, $2 \mathrm{X}$

Troyano Pujadas, I., 2T

Tseng, C. H., $2 \mathrm{H}$

Tucker, C., 11

Tulloch, Simon, 2C, 2Z, 30

Turck, K., 11

Tutt, James H., 06

Tyson, Anthony, $1 \mathrm{~A}$

Uchida, Tomohisa, 10

Ulmer, Melville P., OP

Vadawale, S. V., 2J

Vampola, John, 2X

Van Berg, R., OX

van der Biezen, John, $1 \mathrm{~N}$

van der Luijt, Cornelis, OU, OZ, 1E, 1G, 1N, 21

Van Duinkerken, Gertjan, $1 \mathrm{E}$

Van Winckel, H., IP

Verhoeve, Peter, OU, 0Z, 21

Viale, T., 17

Visser, IVo, OU, 0Z, 1E, 1G, 1N, 21

von Ballmoos, $\mathrm{P}$., $2 \mathrm{H}$

Waczynski, Augustyn, 0Q, 11

Walter, Christopher, IA

Walter, R., 2T

Waltham, Nick, OY, IS

Walton, D., 10

Wang, F., 11

Wang, Jian, 1K, 1L

Wang, Jian-min, $1 \mathrm{~L}$
Wang, Shiang-Yu, 14

Warmbier, Eric A., ON

Weatherill, Daniel P., 06

Weber, C. 11

Wei, Mingzhi, 23

Weller, Harald, OR

Wen, Yiting, $O Q$

Wheaton, Skylar, OP

Więcek, M., 2T

Williams, J., 11

Wilson, Matt, 1D

Wilson-Hodge, Colleen, ID

Wolf, Marsha, $2 \mathrm{U}$

Wollack, Edward, 2V

Wong, Andre, OD

Wood, Daniel, Ol, OJ, OL

Yang, C. Y., 2H

Yelkenci, F. Korhan, 2P

Yerli, Sinan K., 2P

Yeşilyaprak, Cahit, 2P

Yoshida, Michitoshi, 10

Zagdański, A., 2T

Zanatta, Jean-Paul, OC

Zandian, Majid, OF, OW

Zhang, Hong-fei, 1K, $1 \mathrm{~L}$

Zhang, Yuheng, 23

Ziętara, K., 2T

Zoglaver, A., $2 \mathrm{H}$

Żychowski, P., 2T 
Proc. of SPIE Vol. $9915991501-14$

Downloaded From: https://www.spiedigitallibrary.org/conference-proceedings-of-spie on 26 Apr 2023 Terms of Use: https://www.spiedigitallibrary.org/terms-of-use 


\section{Conference Committee}

Symposium Chairs

Colin Cunningham, UK Astronomy Technology Centre (United Kingdom)

Masanori lye, National Astronomical Observatory of Japan (Japan)

Symposium Co-chairs

Allison A. Barto, Ball Aerospace \& Technologies Corporation (United States)

Suzanne K. Ramsay, European Southern Observatory (Germany)

Conference Chairs

Andrew D. Holland, e2v Centre for Electronic Imaging at The Open University (United Kingdom)

James Beletic, Teledyne Imaging Sensors (United States)

Conference Program Committee

Megan E. Eckart, NASA Goddard Space Flight Center (United States)

Gert Finger, European Southern Observatory (Germany)

Michael E. Hoenk, Jet Propulsion Laboratory (United States)

Paul Jorden, e2v technologies plc (United Kingdom)

Didier D. Martin, European Space Research and Technology Centre (Netherlands)

Satoshi Miyazaki, National Astronomical Observatory of Japan (Japan)

Peter C. Moore, National Optical Astronomy Observatory

(United States)

S. Harvey Moseley, NASA Goddard Space Flight Center (United States)

Robert H. Philbrick, Ball Aerospace \& Technologies Corporation (United States)

Roger M. Smith, California Institute of Technology (United States)

Tadayuki Takahashi, Japan Aerospace Exploration Agency (Japan)

Hiroshi Tsunemi, Osaka University (Japan) 
Session Chairs

1 Detector Programmes

Andrew D. Holland, e2v Centre for Electronic Imaging (United Kingdom)

2 EM CCDs

Michael E. Hoenk, Jet Propulsion Laboratory (United States)

3 IR Sensors

James W. Beletic, Teledyne Imaging Sensors (United States)

4 Radiation Damage Studies

Andrew D. Holland, e2v Centre for Electronic Imaging (United Kingdom)

5 IR Detectors I

Gert Finger, European Southern Observatory (Germany)

6 CCDs

Paul R. Jorden, e2v technologies plc (United Kingdom)

7 Test and Characterisation

Nick Nelms, ESA/ESTEC (Netherlands)

8 CMOS Image Sensors

Andrew D. Holland, e2v Centre for Electronic Imaging (United Kingdom)

James W. Beletic, Teledyne Imaging Sensors (United States)

9 QE Improvements and Calibration

Andrew D. Holland, e2v Centre for Electronic Imaging (United Kingdom)

10 CMOS X-ray Sensors

Hiroshi Tsunemi, Osaka University (Japan)

11 IR Detectors II

James W. Beletic, Teledyne Imaging Sensors (United States) 


\section{Introduction}

Astronomical Telescopes and Instrumentation once again combined the visible, infrared, and high energy detector sessions into a single conference; High Energy, Optical, and Infrared Detectors for Astronomy VII. This combination resulted in a rich variety of applications and detectors being presented by the community. This combination also provided an excellent overview of the leverage of detector technologies and methods across the photon energy spectrum, where many of the detection techniques and methodologies are common. Sessions were also held into associated readout electronics and radiation damage which are important for many applications.

Over ninety papers were presented over four days and attendance was high through the conference sometimes exceeding 160 attendees. This reflects on the excellence of presented material, the presenting authors, and the relevance of the conference to present-day astronomy.

The presentations covered detector performance, both theoretical, simulations and experimental, detectors in instruments and camera systems, sophisticated new controllers and software, packaging of very large detector mosaics, radiation testing, and the future direction of sensor technologies.

Over the years, proceedings like these have been an invaluable output and record of SPIE meetings. They represent a current snapshot of detector technologies. This year's conference had several talks on CCDs covering optical and x-ray bands, and included a session on electron-multiplying CCD technology and applications. This year we saw an increase in the number of contributions detailing the continued development and improvement of both CMOS imagers, for the optical, and with new developments for their use in x-ray detection. Many results were "hot off the press", with some being taken just days before the conference, which helps maintain the vitality of these events. We hope the detailed information presented here will contribute to further advancements in all detector technologies.

Such a successful meeting could not have taken place without the support and help of many people, especially all of you whose names appear on the papers collected here. We acknowledge the valuable advice and assistance for structuring the conference and chairing the sessions given to us by the Program Committee and would especially like to thank Jim Beletic, Gert Finger, Paul Jorden, Peter Verhoeve, Michael Hoenk and Hiroshi Tsunemi for their assistance in chairing some of the sessions.

Finally, we hope that you enjoy the written proceedings as an accurate record of the conference, and look forward to seeing you in Austin in 2018.

\section{Andrew D. Holland James Beletic}


Proc. of SPIE Vol. $9915991501-18$

Downloaded From: https://www.spiedigitallibrary.org/conference-proceedings-of-spie on 26 Apr 2023 Terms of Use: https://www.spiedigitallibrary.org/terms-of-use 